\title{
RESTAURACIÓN DE UN PLANO CON SOPORTE DE PAPEL TRANSLÚCIDO REFERENTE A LA "RUTA DEL FERROCARRIL de Mérida a Sisal, YucatáN"
}

Alejandra Odor Chávez*

\section{Resumen}

Dentro del patrimonio documental que resguarda el AGN existe una gran diversidad de soportes, entre ellos, planos y dibujos sobre papeles translúcidos, los cuales son muy sensibles a la humedad y al uso constante, por lo que comúnmente presentan deterioros avanzados. El texto aborda la restauración de un plano de 1866, con la "Ruta del ferrocarril de Mérida a Sisal, Yucatán", sobre un soporte de papel translúcido. Se presentan los antecedentes, las características y estado de conservación del plano, así como la experiencia durante su restauración, que significó un gran reto por sus dimensiones y deterioro. Se resalta el intercambio de información entre los restauradores y el investigador interesado en consultar el plano, pues fue muy enriquecedor para ambas partes.

Palabras clave: papel translúcido, conservación y restauración, laminado.

\begin{abstract}
Within the documentary heritage that protects the AGN there is a wide variety of supports, including plans and drawings on tracing paper, which are very sensitive to dampness and constant use, so commonly present advanced deterioration. The text addresses the restoration of a 1866 plane, with path railroad Mérida Sisal, Yucatán, on a tracing paper support. Background, characteristics and condition of the plane, and the experience during its restoration, which meant a big challenge because of its size and deterioration are presented. It highlights the exchange of information between restaurateurs and
\end{abstract}

\footnotetext{
* Jefa del Departamento de Conservación y Restauración del AGN.
} 
the researcher interested in viewing the plane because it was very rewarding for both parties.

Keywords: Tracing paper, Restoration and conservation, Leaf casting.

\section{Introducción}

El Archivo General de la Nación (AGN) resguarda parte importante de la memoria documental nacional, cuenta con un acervo que supera los 52 $\mathrm{km}$ lineales. El patrimonio resguardado consta de material bibliográfico, hemerográfico, fotográfico (tanto negativos como positivos) y audiovisual, así como diversos tipos de documentos con soporte de papel, entre ellos una gran cantidad de planos y dibujos sobre papel calco, ${ }^{1}$ comúnmente llamado "transparente", aunque las técnicas de manufactura y las características de éstos son muy variadas de acuerdo a su temporalidad y uso original.

Bringas y Castañeda proponen en su investigación referirse a este tipo de papeles como papeles translúcidos, ${ }^{2}$ por las cualidades ópticas de los mismos a partir de la dispersión de la luz entre sus fibras, y para fines de homologación de términos entre colegas, así serán llamados a lo largo de este texto.

Por sus materiales constitutivos y técnicas de manufactura, los papeles translúcidos suelen ser sumamente sensibles a la humedad directa, e incluso a las fluctuaciones de las condiciones ambientales (especialmente la humedad relativa). Comúnmente el tipo de obra realizada sobre estos soportes es de gran formato (fueron muy utilizados para trazar planos y/o para hacer calcas a escala), por lo cual su manipulación y almacenamiento representan riesgos adicionales a los que sus materiales constitutivos les confieren.

1 Papel calco es la traducción del término en inglés tracing paper. Jennifer Libertad Bringas y María Magdalena Castañeda, "Análisis de las propiedades físico mecánicas del adhesivo hidroxipropilcelulosa en el proceso de laminado de documentos en soporte de papel albanene y vegetal", México, 2013, tesis, ENCRyM, p.11.

2 Libertad Bringas y María Magdalena Castañeda, "Análisis de las propiedades físico mecánicas del adhesivo hidroxipropilcelulosa en el proceso de laminado de documentos en soporte de papel albanene y vegetal”, México, 2013, tesis, ENCRyM, p. 26. 
Debido a lo anterior, resulta común encontrar acervos documentales con soportes de papel translúcido, que presentan deterioros como rigidez, fragilidad, marcas de dobleces, roturas en las orillas (especialmente si se almacenan enrollados), roturas y faltantes asociados a dobleces, y deformaciones por contacto directo con humedad y/o fluctuaciones en las condiciones ambientales.

Este texto aborda la restauración de un plano en papel translúcido de gran formato, perteneciente a la Colección de Mapas, Planos e Ilustraciones (Mapilu) del AGN, mediante la utilización de la técnica de laminado con adhesivos reactivados con solventes, ${ }^{3}$ exponiendo las dificultades, retos y beneficios obtenidos a partir de la aplicación de una técnica cada vez más conocida, pero relativamente poco aplicada, para la restauración de obra documental susceptible a la humedad o a ciertos tratamientos acuosos.

\section{El plano a restaurar: "Ruta del ferrocarril de Mérida a Sisal, Yucatán”}

El plano intervenido es un registro de la "Ruta del ferrocarril de Mérida a Sisal, Yucatán" del año 1866. ${ }^{4}$ Debido a su mal estado de conservación y considerable fragilidad, no había sido descrito y mucho menos digitalizado, no se conocían sus dimensiones, y únicamente se contaba con el título, mismo que resultaba relativamente visible por estar localizado en uno de los fragmentos superiores del documento.

A raíz de las constantes peticiones de un investigador, quien solicitó la consulta del plano como parte importante de su tema de estudio, (relacionado con el autor del plano, el ingeniero Ignacio Fernández de Galindo), se consideró la posibilidad de estabilizar la obra para obtener una imagen digital y permitir de este modo su consulta.

3 Un laminado consiste en la adhesión de un papel japonés en la totalidad de una de las caras del documento, para recuperar su estabilidad mecánica cuando presenta demasiadas roturas, faltantes o fragilidad considerable.

4 AGN, Mapas, Planos e Ilustraciones, número 3760. 


\section{Características generales}

Se trata de un plano de aproximadamente $248 \times 68 \mathrm{~cm}$, conformado a partir de la unión de cuatro pliegos de papel translúcido, cuya gran transparencia se obtuvo a partir de la impregnación del papel en alguna resina o aceite. ${ }^{5}$ Debido a esto, la sensibilidad del soporte ante el agua no es tan marcada como lo sería en un papel albanene o vegetal, cuya transparencia se obtiene por medios mecánicos, como el ultra refinado de las fibras o el súper calandrado de la hoja, ${ }^{6}$ pero tampoco resultaba posible la aplicación directa de humedad sin ocasionar deformaciones importantes en el papel, que además es sumamente delgado y transparente.

Por otro lado, las tintas presentes en el plano (negra y roja) son solubles en agua y ligeramente solubles en alcohol, lo que limitaba aún más los posibles tratamientos para su estabilización física.

Durante el diagnóstico del plano se detectó una marca de producción del soporte la cual para el investigador significó una importante aportación, al ubicar regionalmente el papel utilizado por el ingeniero Fernández de Galindo. En uno de los pliegos, se encontró un gofrado con el nombre Angoulême, una región en Francia, conocida por el auge de su producción papelera durante el siglo XIX, y en donde actualmente existe un Museo del Papel. ${ }^{7}$

\section{Estado de conservación}

Debido a que el plano estuvo almacenado en una guarda de tamaño similar al formato carta, se encontraba doblado en al menos 20 partes, y casi en todos los casos el doblez ya había generado la ruptura del soporte en dichas zonas, dejando más de 40 fragmentos.

Aunado a la fragmentación, el plano presentaba una oxidación y fragilidad considerable y, al ser muy delgado, era prácticamente imposible

5 Durante la intervención de restauración, en ocasiones se detectaba un ligero aroma parecido al de la resina colofonia, por lo que probablemente el soporte fue impregnado con dicha resina o algún material similar.

6 Bringas y Castañeda, op. cit., pp. 34-38.

7 Musée du papier d'Angoulême en <http://www.angouleme.fr/museep/> [consulta: agosto 2012]. 
manipularlo sin ayuda de un soporte auxiliar, aun cuando se trataba de fragmentos pequeños.

Dicho deterioro es típico de papeles translúcidos de este tipo: "El papel translúcido que se obtiene por la adición de sustancias como aceites y resinas, aunado a las condiciones del medio ambiente, se vuelve más frágil, quebradizo, y amarillento con el tiempo". ${ }^{8}$ El soporte de papel también presentaba algunas deformaciones, probablemente por fluctuaciones en las condiciones ambientales, así como dobleces y faltantes.

Los elementos plasmados se encontraban en buen estado, pero su principal problema era la manipulación del soporte, pues en esas condiciones era prácticamente imposible desdoblarlo y/o extenderlo para consultarlo o digitalizarlo sin ocasionarle mayores daños al documento.

\section{Antecedentes}

Desde 2010, debido a la necesidad de restaurar diversas obras sobre distintos soportes de papel translúcido, relacionadas con el pabellón de México en la Exposición Universal de 1900, el Departamento de Conservación y Restauración del AGN consideró la necesidad de implementar alguna técnica que permitiera el refuerzo estructural de dichos documentos, aportando la menor cantidad de agua posible durante los tratamientos, para limitar el riesgo de causar deformaciones y/o modificar las dimensiones de los documentos tratados, pues suelen ser planos a escala.

Se realizaron pruebas con probetas de papel albanene moderno, comparando los resultados obtenidos a partir del laminado con papel japonés y adhesivos tradicionales en la restauración de papel como la metil celulosa y el almidón de trigo, además de la técnica de reactivado de Klucel G con alcohol y alcohol-acetona, ${ }^{9}$ propuesta por Susan Page desde $1997 .{ }^{10}$

8 Bringas y Castañeda, op. cit., p. 43.

9 El Klucel G es un adhesivo a base de hidroxipropil celulosa, utilizado en la restauración de papel por su compatibilidad con el mismo, y por su estabilidad y reversibilidad a largo plazo. 10 Susan Page, Conservation of Nineteenth-Century Tracing Paper: A Quick Practical Approach, en http://cool.conservation-us.org/coolaic/sg/bpg/annual/v16/bp16-09.html, [consulta: marzo 2010]. 
En dichas pruebas, tanto en flexibilidad, como en la transparencia obtenida después del laminado (este es un punto sumamente importante a considerar durante la restauración de papeles translúcidos), el laminado con Klucel $G$ reactivado con alcohol etílico presentó los mejores resultados. En cuanto a poder adhesivo, en todos los casos se consiguió una buena adhesión entre el papel de la probeta y el papel japonés del laminado.

Con base en las pruebas realizadas, se decidió llevar a cabo el laminado con Klucel $\mathrm{G}$ reactivado con alcohol etílico, en planos de papel albanene e impregnados con resinas, de mediano formato; se obtuvieron óptimos resultados, ${ }^{11}$ así como algunas intervenciones menores (refuerzos de papel japonés) en diversas obras de papel vegetal, albanene, y papeles impregnados, con lo que se constató el comportamiento de la técnica en diversos tipos de soporte translúcido.

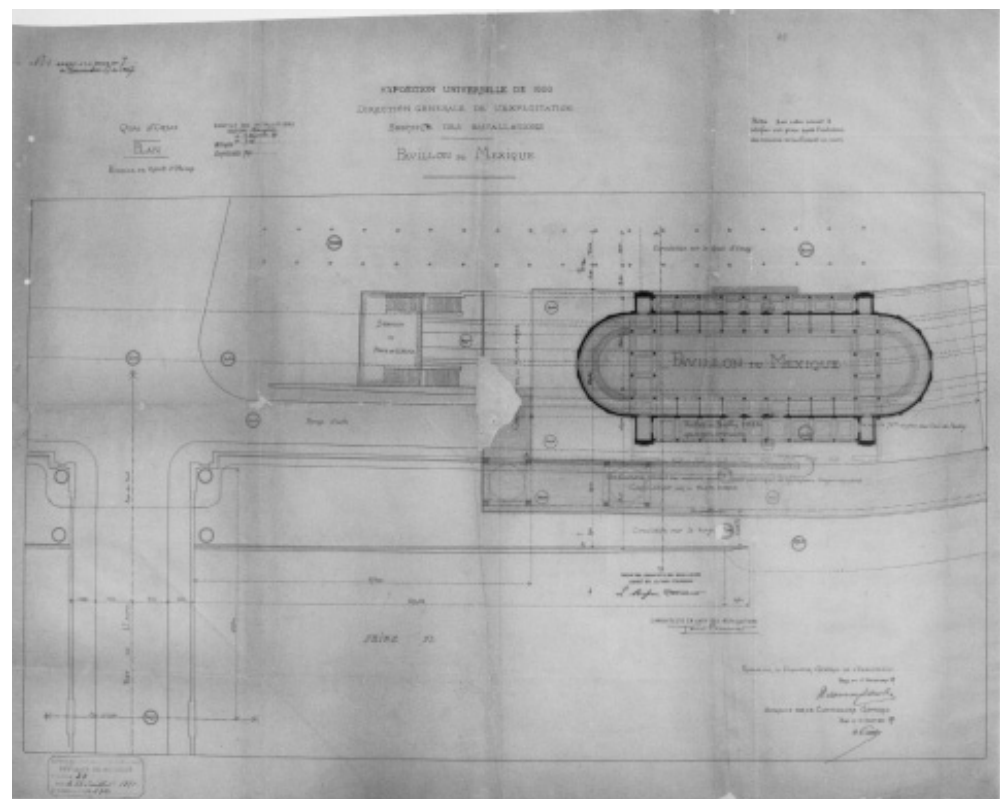

Plano de papel translúcido impregnado, laminado con Klucel $G$ reactivado con alcohol etílico, después de la intervención de restauración

11 Durante estas pruebas se hicieron algunas adaptaciones a la técnica propuesta por Page -quien utiliza la mesa de succión durante el tratamiento-, pues en el Departamento de Conservación y Restauración del AGN no se contaba con una mesa de succión. 
La técnica de Klucel $G$ reactivado con solventes se ha empleado para papeles sensibles a la humedad, en material fotográfico, e incluso actualmente se considera como una de las mejores opciones para la estabilización mecánica de ciertos documentos con tintas ferrogálicas con exceso de iones metálicos, ${ }^{12}$ pues brinda estabilidad física al soporte dañado por corrosión de la tinta, ${ }^{13}$ y durante el tratamiento prácticamente no hay aporte de humedad, lo cual podría ser perjudicial en el caso de este tipo de tintas ferrogálicas.

A grandes rasgos, la técnica consiste en preparar un papel japonés del grosor deseado, con varias capas de Klucel G en una de sus caras, dejándolas secar por completo y posteriormente son reactivadas, por lo general con cantidades mínimas de alcohol etílico (o mezclas de alcohol-acetona).

Debido a los buenos resultados obtenidos en la serie de documentos de la Exposición Universal de 1900, así como en tratamientos locales en documentos con tintas ferrogálicas con exceso de iones metálicos, la técnica de Klucel $G$ reactivado con alcohol etílico se consideró como una buena opción para el tratamiento del plano de la "Ruta del ferrocarril de Mérida a Sisal, Yucatán”, el cual por sus materiales constitutivos y técnica de manufactura representaba ciertas limitantes en cuanto a la aplicación de tratamientos acuosos, pero que requería una estabilización mecánica para poder ser digitalizado y consultado.

\section{Propuesta inicial}

Debido a la carga de trabajo y a la reducción del personal en el Departamento de Conservación y Restauración, así como a la gran inversión de tiempo y recursos materiales que la restauración del plano en cuestión significaría, la primera opción a considerar fue desdoblar y extender los fragmentos, ubicar su posición, estabilizarlos mediante pequeños refuerzos (con papel

12 Eliza Jacobi, et al., "Rendering the invisible visible, preventing solvent-induced migration during local repair on iron gall ink", pp. 25-34.

13 El término corrosión de la tinta se utiliza para denominar al deterioro comúnmente causado por tintas ferrogálicas con exceso de iones metálicos en su composición, y que se observa como halos alrededor de los trazos, trasminación del texto e incluso carbonización del soporte de papel. Las tintas ferrogálicas fueron las tintas más utilizadas para manuscritos en México desde la colonia y hasta inicios del siglo xx. 
japonés previamente preparado con Klucel $G$ para ser reactivados), y digitalizar el plano, para permitir su consulta mediante la imagen obtenida, de manera tal que, posteriormente, el plano pudiera almacenarse extendido en la Mapoteca del AGN.

Se procedió al registro fotográfico del documento y a la separación de los fragmentos que se encontraban apilados uno sobre otro, utilizando láminas de Mylar ${ }^{14}$ debajo de cada fragmento para darle soporte y poder transportarlo con seguridad al momento de armar el plano. Fue hasta ese momento cuando se conocieron las dimensiones del documento y la cantidad de faltantes que presentaba.

El proceso de ubicar los fragmentos para armar el plano fue complejo, pues la mayoría del camino trazado se encontraba en blanco. Para este fin, fue de gran ayuda la colaboración del investigador, quien solicitó la consulta del documento, ya que nos proporcionó varios mapas actuales de la ruta trazada en el plano.

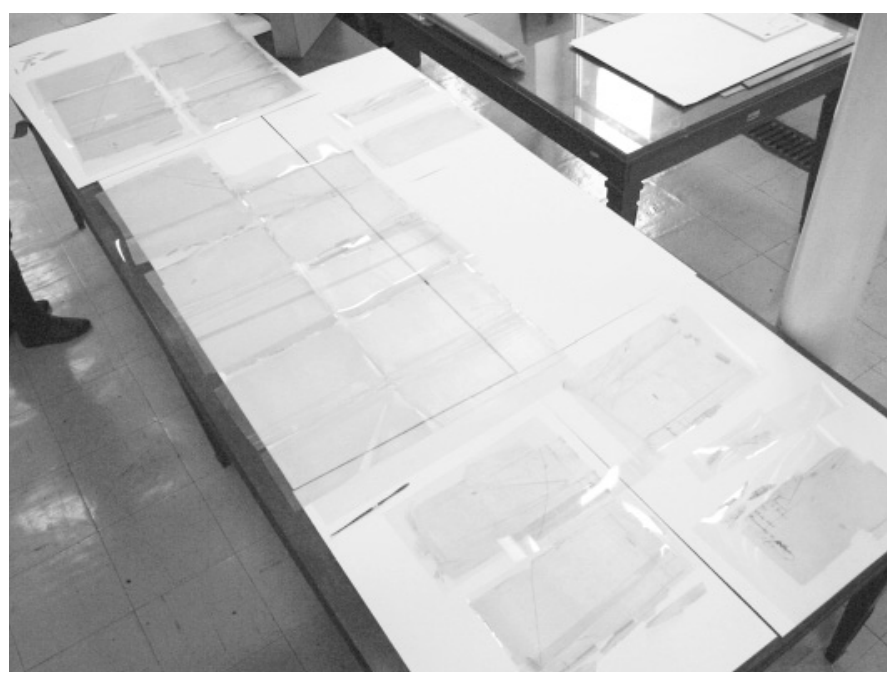

Algunos de los fragmentos del plano sobre soportes de Mylar para facilitar su movimiento y ubicación

14 Lámina delgada de poliéster transparente. 

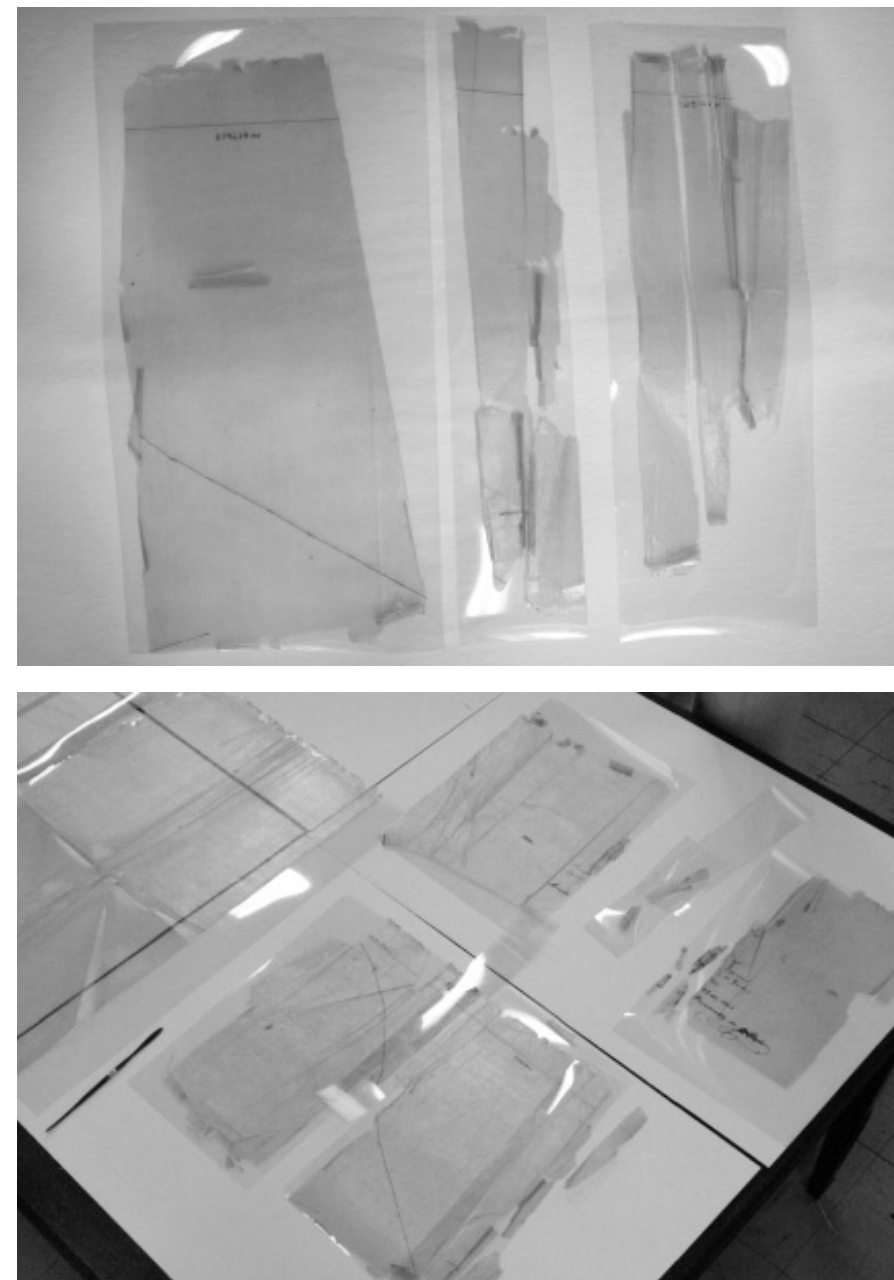

Algunos de los fragmentos del plano sobre soportes de Mylar para facilitar su movimiento y ubicación

De manera simultánea se fue estabilizando cada fragmento por separado, desdoblando con ayuda de pinzas y espátulas las partes dobladas y encimadas, recuperando el plano (en la medida de lo posible y únicamente mediante la aplicación de alcohol y ligera presión), y colocando pequeños refuerzos de papel japonés previamente preparado con Klucel G, y reactivados con alcohol etílico para adherirlos. Estos refuerzos se colocaban de forma 
seccionada, y únicamente en aquellas zonas consideradas como riesgosas para la manipulación del plano durante su digitalización.

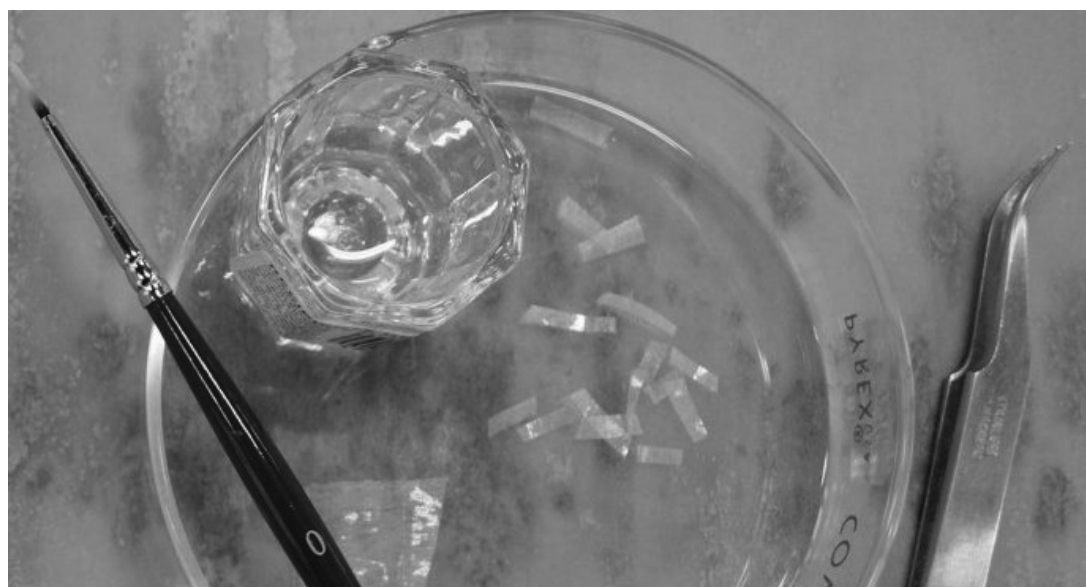

Detalle de refuerzos de papel preparado con Klucel G para reactivar, similares a los utilizados en el plano

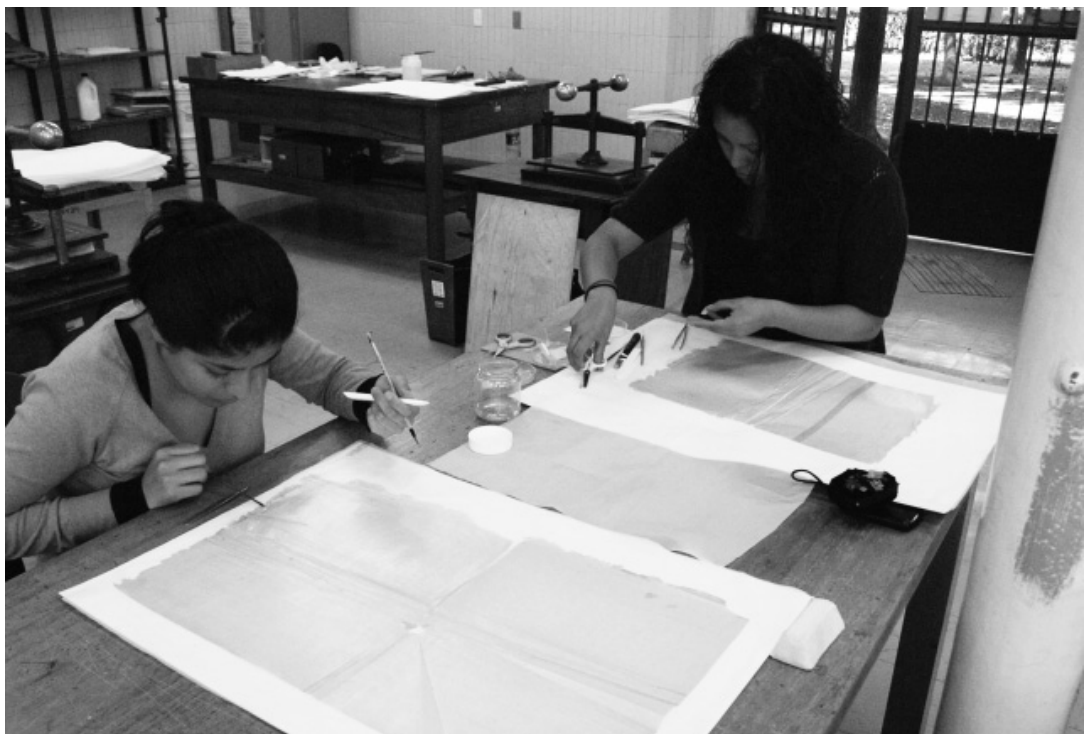

Proceso de estabilización de los fragmentos del plano, mediante la aplicación de refuerzos de papel japonés preparado con Klucel G 


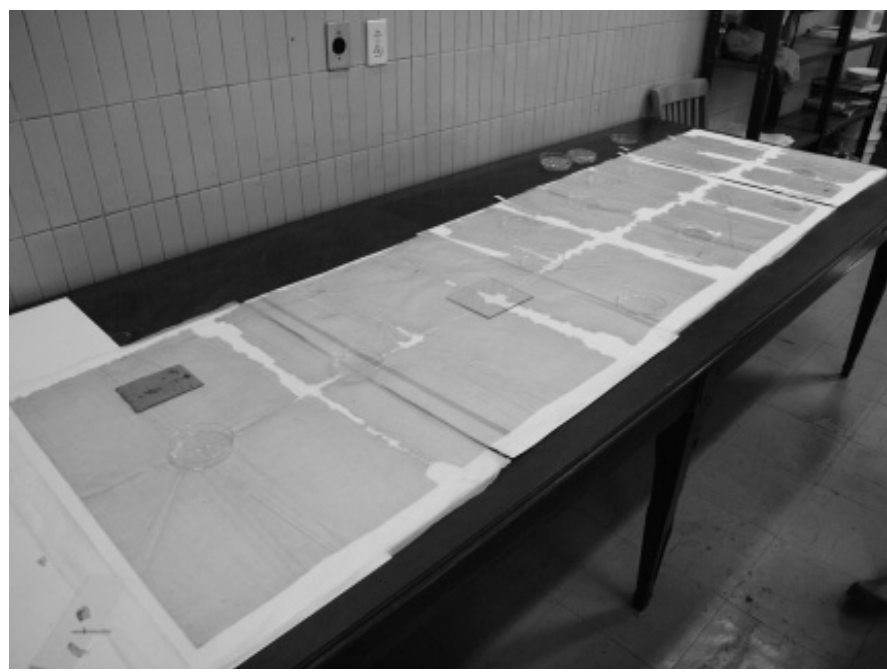

Estabilización de fragmentos

Cuando se tuvieron todos los fragmentos estabilizados y ubicados, comenzaron a unirse entre sí con la misma técnica. Al notar la existencia de demasiados faltantes en las zonas de unión entre los ellos, se puso en duda que los refuerzos propuestos fueran suficientes para mantenerlos unidos a largo plazo, e incluso que resistieran la digitalización, pues por las dimensiones de la obra, ésta debería llevarse a cabo por partes. Por otro lado, y debido a la gran fragilidad del soporte y al espacio destinado en la Mapoteca del AGN para su resguardo, la idea de almacenarlo extendido tampoco resultaba ya la opción más viable.

\section{Propuesta final}

Debido a lo expuesto en el apartado anterior, y con base en los resultados obtenidos en laminados previos de papel translúcido con la técnica de Klucel $\mathrm{G}$ reactivado con alcohol etílico, ${ }^{15}$ se decidió brindarle mayor soporte al plano y unir los fragmentos mediante un laminado, el cual inicialmente no estaba contemplado.

15 Remitirse al apartado de antecedentes. 
Se adquirió un papel japonés ultra delgado $\left(5 \mathrm{gr} / \mathrm{m}^{2}\right){ }^{16}$ para que aun con el laminado, el plano conservara la gran translucidez que tuvo de origen, y por las dimensiones del mismo se decidió laminarlo por partes, es decir adhiriendo cada pliego de papel japonés por separado, siendo necesarios cuatro pliegos.

Se prepararon los cuatro pliegos de papel japonés, con tres capas de Klucel $G$ al 3\% en agua, de acuerdo con la técnica de Susan Page, ${ }^{17}$ y posteriormente se fueron adhiriendo uno a uno al plano que ya se encontraba completamente armado y sujeto mediante los refuerzos de papel japonés aplicados previamente.

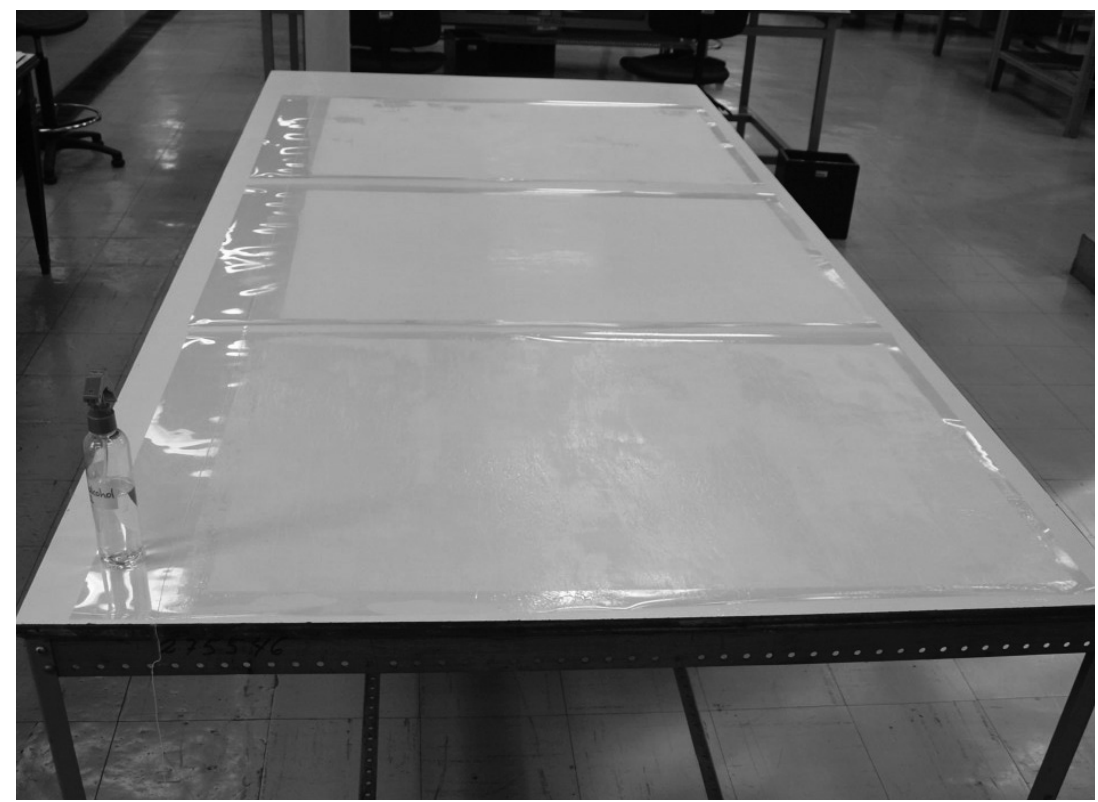

Preparación del papel japonés para el laminado con Klucel G reactivado

16 Papel fabricado en Japón, 100\% de fibras de kozo, que son muy largas, estables y resistentes. 17 Susan Page, op. cit. 
El proceso consistió en colocar el papel japonés con las capas de Klucel $G$ hacia el documento, y comenzar a aplicar alcohol etílico mediante aspersión, mientras se pasaba una brocha seca sobre el papel de laminado para asegurar el contacto con el plano. Al tratarse de un papel japonés tan delgado, la cantidad de alcohol requerida para reactivar el adhesivo fue mínima.

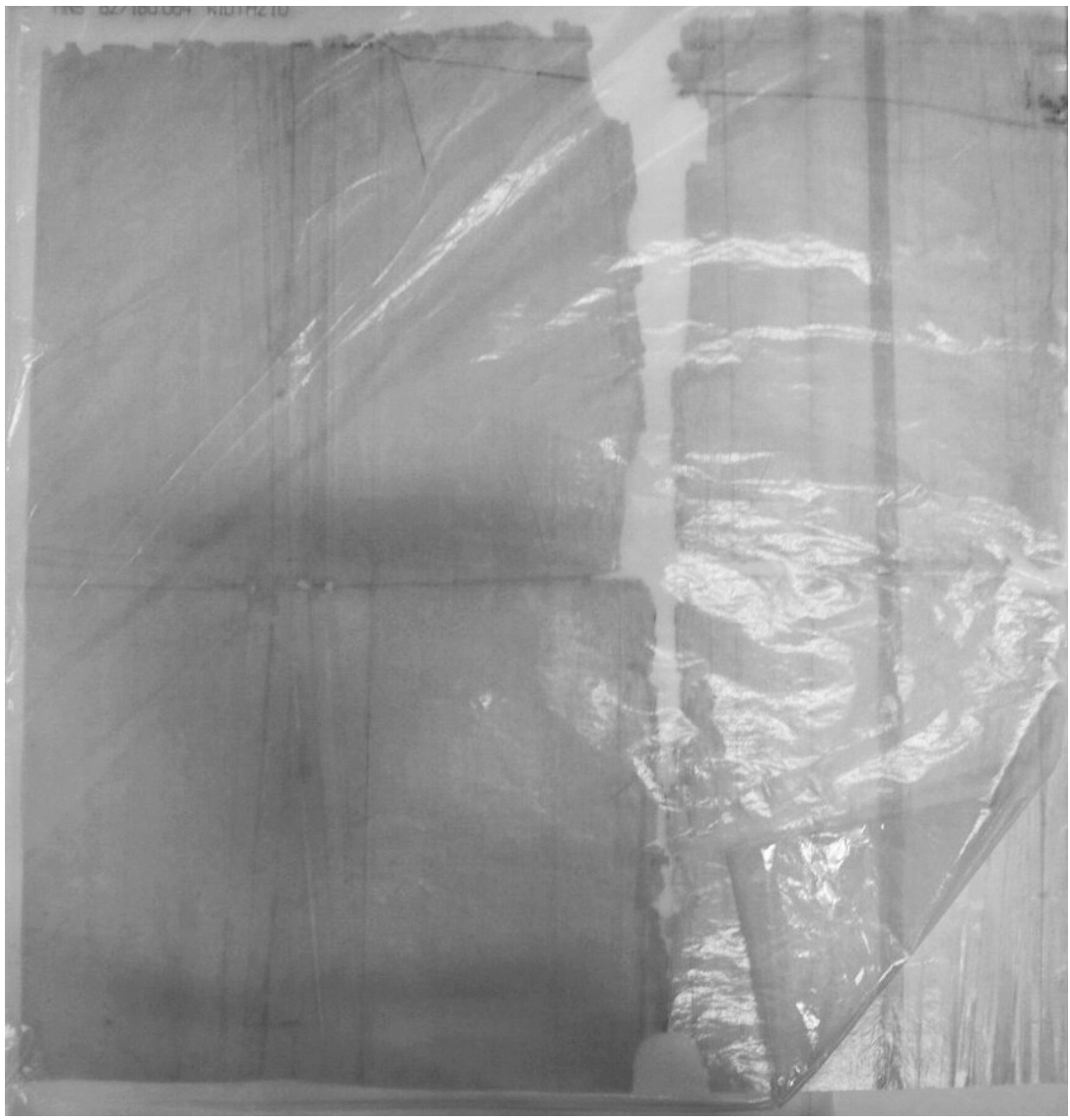

Papel japonés preparado con Klucel G, sobre el plano a laminar 
Posteriormente se colocaba peso y se dejaba secar al menos un día entre la adhesión de cada pliego. En algunas ocasiones se tenían zonas con menor adhesión entre los soportes, lo cual se solucionaba fácilmente, aplicando alcohol de forma local y volviendo a secar bajo peso.

Durante el tratamiento de este plano, y en general al aplicar la técnica de Klucel $G$ reactivado con alcohol etílico, ha sido evidente la gran flexibilidad que los soportes laminados adquieren después del tratamiento, y la importancia del peso durante el secado del mismo, pues en la mayoría de las ocasiones la falta de adhesión en algunas zonas, se relacionaba con la falta de peso o con la distribución heterogénea de este.

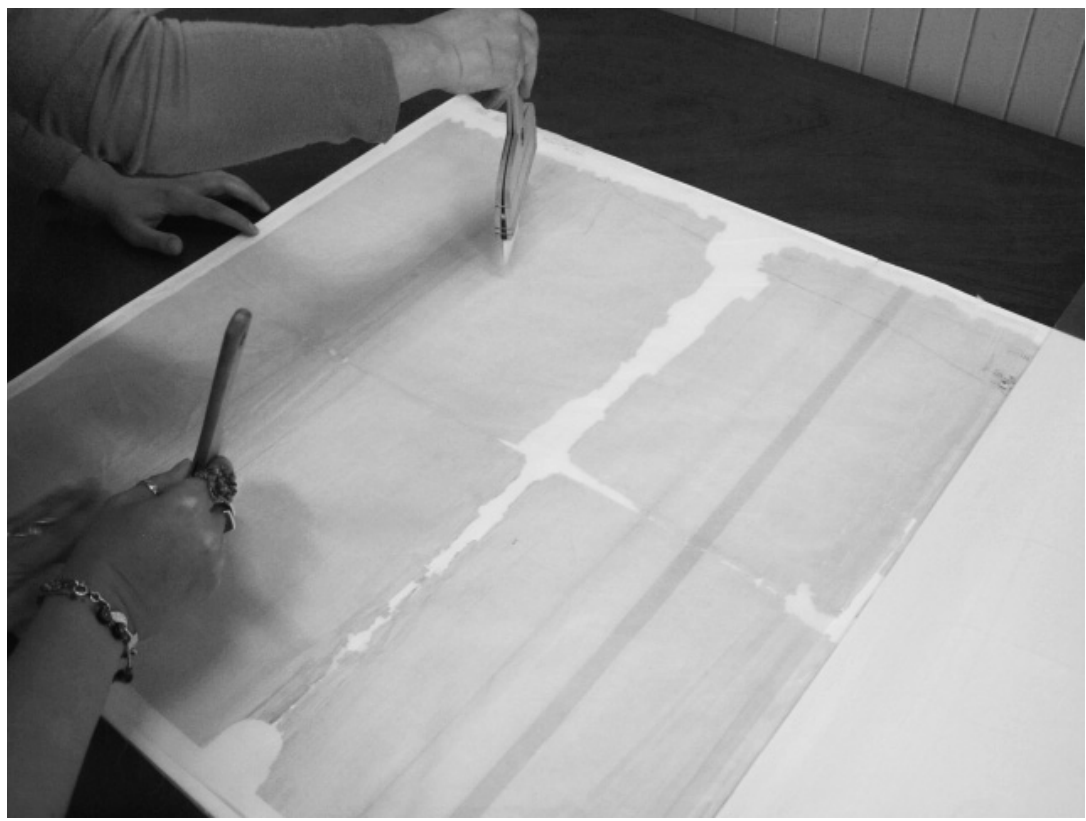

Proceso de reactivación del adhesivo y unión entre ambos soportes de papel 
Una vez finalizado el proceso de laminado se consideró que la intervención le brindaba al documento la estabilidad suficiente para ser digitalizado y almacenado con seguridad (los objetivos iniciales de la intervención), además de no resultar prioritaria la colocación de injertos, ${ }^{18}$ los cuales si bien le hubieran brindado mayor estabilidad y unidad visual al plano, implicarían una gran inversión de recursos humanos y materiales no contemplados y que ya habían sido rebasados conforme a la propuesta original.

De este modo, se decidió digitalizar el plano al concluir el laminado y buscar una forma de almacenamiento para brindarle protección a largo plazo, pero que también resultara viable en cuanto a recursos materiales y al espacio disponible para resguardarlo, tomando en cuenta que la consulta del original estará restringida al quedar disponible para el usuario la imagen digital.

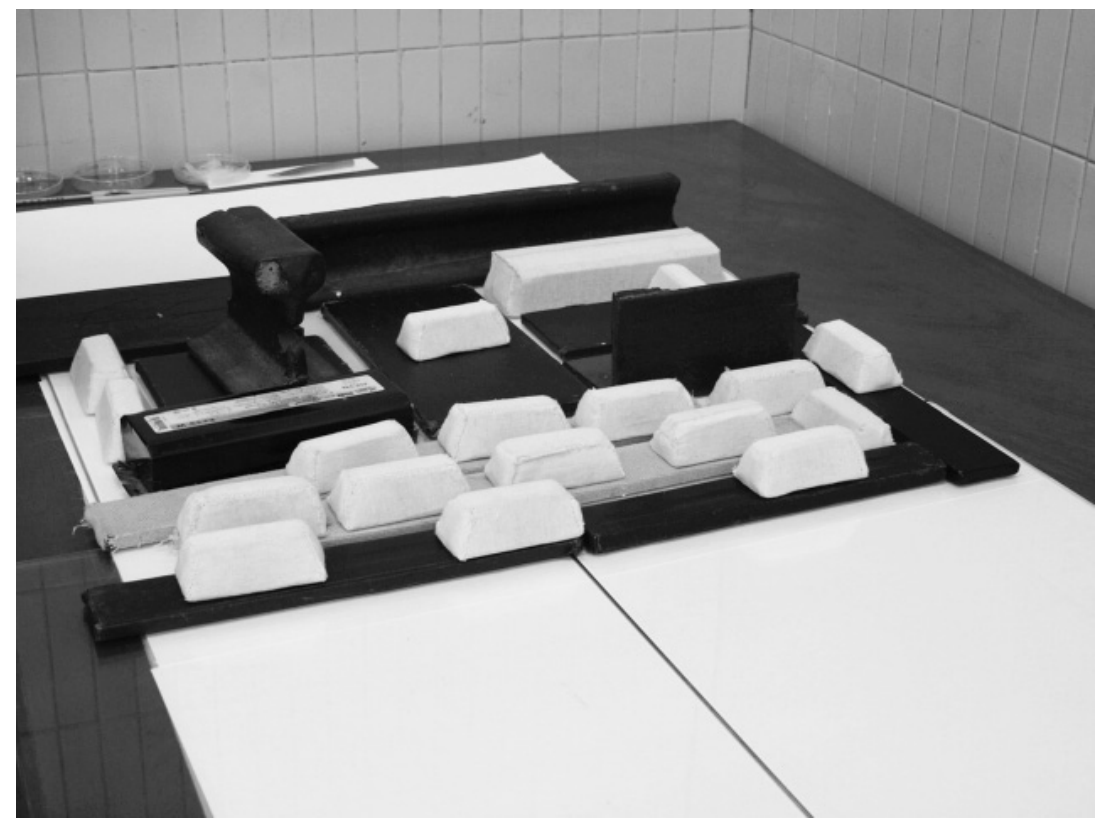

Colocación de peso sobre la zona del plano que fue laminada

18 Los injertos son fragmentos de papel japonés de grosor similar o igual al del documento restaurado, que se colocan en las zonas de faltantes de papel original, para brindarle mayor estabilidad mecánica y unidad visual al documento. 


\section{Almacenamiento del plano}

Con base en lo anterior, se aprovechó la gran flexibilidad que el laminado con Klucel $G^{\circledR}$ le brindó al papel original, esto permitió desarrollar un sistema de montaje para almacenar el plano enrollado, minimizando el espacio a ocupar en las áreas de resguardo, lo cual en un acervo de las dimensiones del AGN resulta prioritario y necesario en muchas ocasiones.

Si bien la forma ideal de almacenar obra de gran formato es extendida, también es posible almacenarla enrollada, siempre y cuando se le brinde soporte y protección para evitar los deterioros típicos del material enrollado.

Se optó por enrollar el plano alrededor de un tubo de cartón de $20 \mathrm{~cm}$ de diámetro, a modo de soporte o "alma", para evitar el daño de la obra ante el movimiento o cualquier golpe. Para esto, y debido al presupuesto limitado con el que se contaba, se reutilizó un tubo vacío (previamente utilizado para almacenar un rollo de papel libre de ácido), éste se forró con papel Tyvek. ${ }^{19}$ Posteriormente se colocó el plano sobre una tira de papel Tyvek y se protegió en la parte superior con Mylar, permitiendo así la apreciación del plano sin necesidad de tocarlo.

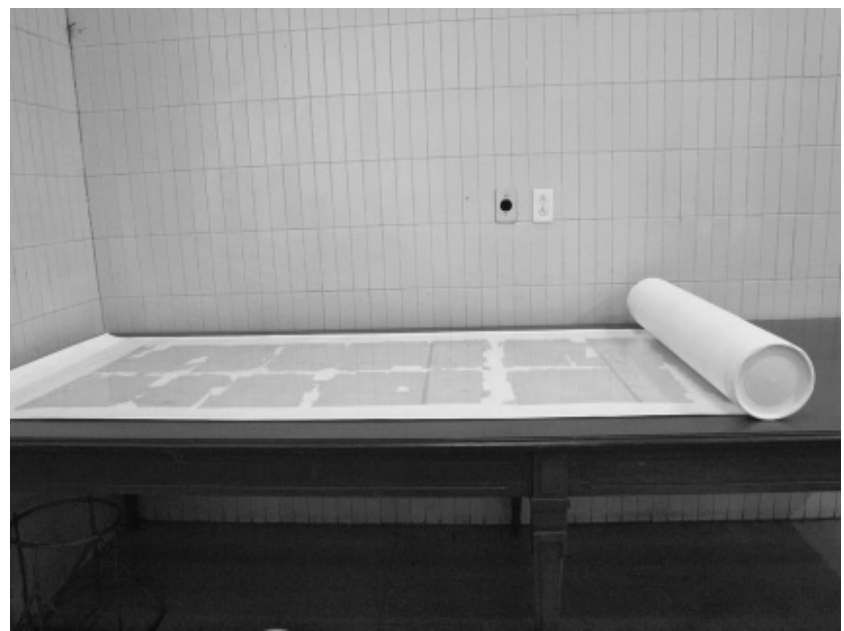

Preparación del tubo y el montaje del plano para su almacenamiento

19 Material 'no tejido' hecho a base de fibras de polietileno de alta densidad, sumamente estable y libre de ácido. 
El plano, entre el Tyvek y el Mylar, se enrolló cuidadosamente sobre el tubo y se sujetó mediante cinta blanca de algodón, de $2 \mathrm{~cm}$ de ancho. En la parte exterior del rollo se colocaron los datos de identificación del plano, así como una fotografía del mismo, para facilitar su ubicación, sin tener que desenrollarlo.

\section{Conclusiones}

La restauración del plano de la "Ruta del ferrocarril de Mérida a Sisal, Yucatán" representó un gran reto para el Departamento de Conservación y Restauración del AGN por diversos factores como: la sensibilidad y fragilidad de sus materiales constitutivos, la gran cantidad de fragmentos y faltantes ocasionados por el sistema previo de almacenamiento, las dimensiones desconocidas en un inicio y que superaron los dos metros de largo, el limitado presupuesto para su restauración y las características del espacio destinado para su resguardo.

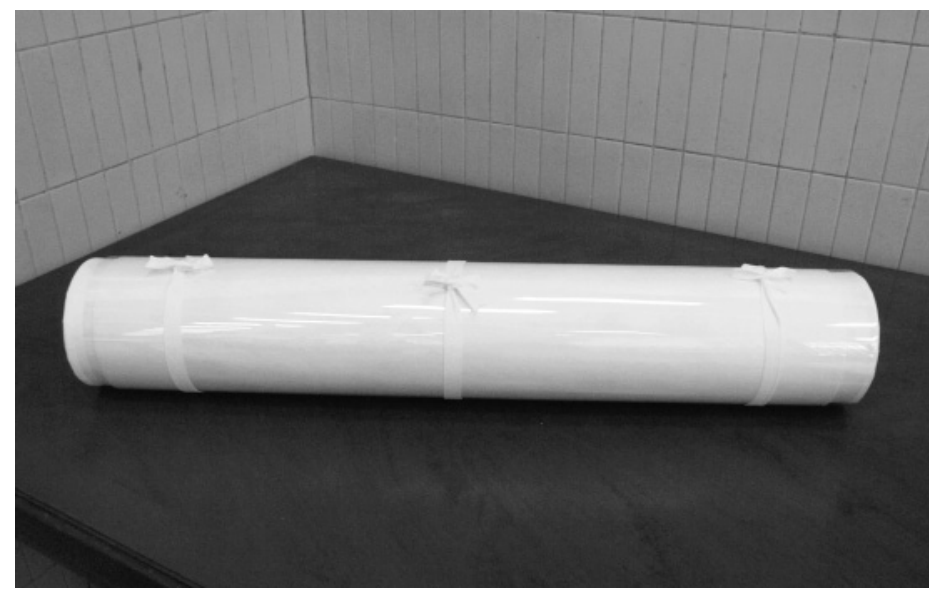

Plano restaurado y enrollado, listo para ser almacenado

La intervención previa de documentos con características similares, así como las pruebas realizadas sobre probetas de papel albanene facilitaron la toma de decisiones durante la restauración del plano, las cuales fueron cambiando conforme se resolvían los problemas detectados inicialmente. 
Este caso es un claro ejemplo de que la propuesta de restauración planteada a partir del diagnóstico inicial de una obra puede cambiar conforme se lleva a cabo la intervención, con la finalidad de conseguir los objetivos planteados originalmente.

La técnica de laminado con Klucel $G$ reactivado con solventes, para el tratamiento de obra sensible ante métodos acuosos, se reporta en publicaciones de al menos hace 16 años; actualmente se aplica en distintos materiales como papel, fotografía e incluso textiles; sin embargo, aún no es un proceso que resulte tan conocido o se aplique de manera común en muchos talleres de restauración, aunque sus resultados en la mayoría de los casos son sumamente favorables.

De este modo, resulta necesario continuar estudiando esta técnica para conocer su estabilidad a largo plazo, mientras se difunde y aplica en diversos casos, compartiendo con otros colegas los resultados obtenidos. En los tratamientos llevados a cabo en el Departamento de Conservación y Restauración del AGN, mediante el reactivado de Klucel G con alcohol etílico, ha sido evidente la importancia del peso al momento del secado del documento para conseguir una buena adhesión entre los soportes. También destaca la gran flexibilidad adquirida por los papeles intervenidos después del laminado (incluso papeles de pulpas mecánicas de madera sumamente quebradizos), la facilidad de aplicación del tratamiento y su reversibilidad a corto plazo (la reversibilidad a largo plazo es una de las múltiples líneas de investigación sobre el tema).

No se trata de una técnica que sustituya a los laminados tradicionales con adhesivos acuosos como la metil celulosa y el almidón de trigo, sino de una buena alternativa para aquellos casos en los cuales la obra requiere una estabilización mecánica, pero debido a diversas razones es sumamente susceptible ante tratamientos acuosos, como lo sería un laminado tradicional. Como en cualquier tratamiento de restauración, es imprescindible distinguir aquellos casos en que resulta una opción viable y necesaria.

Cabe resaltar la importancia de la colaboración del Departamento de Conservación y Restauración con otras áreas del AGN (Control de Acervos Históricos, Centro de Información Gráfica y Digitalización) para asegurar la conservación del plano en cuestión, así como la retroalimentación con el investigador interesado en la consulta del mismo. En un archivo vivo como 
el AGN, este intercambio de información con el usuario de la documentación es sumamente enriquecedor para ambas partes y para la conservación del patrimonio que resguardamos.

También resulta necesario agradecer a la Fundación del AGN por el material aportado para la restauración del plano, así como al personal del Departamento de Conservación y Restauración que participó en esta intervención, especialmente a Alexandra Samkova, Giselle Arcos y Maribel Arroyo, quienes colaboraron de forma voluntaria y permitieron concluir la restauración en un tiempo que resultara viable para la institución.

\section{Referencias}

Bringas Botello, Jennifer Libertad y María Magdalena Castañeda Hernández, "Análisis de las propiedades físico mecánicas del adhesivo hidroxipropilcelulosa en el proceso de laminado de documentos en soporte de papel albanene y vegetal", México, 2013, tesis, ENCRyM.

Jacobi, Eliza, et al., "Rendering the invisible visible, preventing solventinduced migration during local repair on iron gall ink", en Journal of Paper Conservation, vol. 12, núm.2, 2011, pp. 25-34.

Musée du papier d'Angoulême en <http://www.angouleme.fr/museep/> [consulta: agosto 2012].

Page, Susan, Conservation of Nineteenth-Century Tracing Paper: A Quick Practical Approach, en <http://cool.conservation-us.org/coolaic/sg/bpg/ annual/v16/bp16-09.html>, [consulta: marzo 2010]. D 